\title{
Morphometric Studies of Chloroquine-Induced Changes in Hepatocytic Organelles in the Rat ${ }^{1}$
}

\author{
Ann Marie Wisner-Gebhart, Roberta K. Brabec and Robert H. Gray \\ Department of Environmental and Industrial Health. Environmental Cellular Chemistry \\ Laboratory, The University of Michigan, Ann Arbor, Michigan 48109
}

Received January 17, 1980, and in revised form March 14, 1980

\begin{abstract}
The autophagic process is thought to occur both normally and at an accelerated rate in response to many types of stress. The clinical drug chloroquine was a potent inducer of this process when administered intraperitoneally to adult, male rats. Morphometric analysis was used to quantitate the number and volume of autophagic vacuoles, mitochondria, peroxisomes, and lysosomes within hepatocytes of unexposed and drug-exposed rats. Within $2 \mathrm{hr}$ of chloroquine treatment, the number of autophagic vacuoles reached an incidence more than 600 times that observed in controls. In these same hepatocytes, the numbers and volumes of the mitochondria, peroxisomes, and lysosomes also varied from controls and could be correlated with the autophagic process. The results established that the subcellular organelle pattern changed within $6 \mathrm{hr}$ after chloroquine exposure and suggested that the autophagic vacuole is pivotal to the cell's response.
\end{abstract}

\section{INTRODUCTION}

Under various unrelated stress conditions the cellular autophagic process can be induced. Autophagy is a process in which a cell sequesters and degrades portions of its cytoplasm and cytoplasmic organelles within intracellular membranes (Ericsson, 1969; Holtzman, 1973). This process occurs in normal cells and has been implicated with organelle turnover (Ericsson, 1969). The induction of cellular autophagy in response to numerous types of physical, biological, and chemical agents is of interest because of its potential importance in the accommodation of stress in biological systems. The formation of autophagic vacuoles (AV) seems to be a ubiquitous response and has been found in prokaryotes (Leedale and Buetow, 1976) as well as numerous eukaryotic cell types (Holtzman, 1973).

The primary objective of this morphometric study is to make quantitative comparisons of $\mathrm{AV}$ and other organelles in control and chloroquine-treated hepatocytes (Wisner-Gebhart et al., 1979). This drug has been used clinically as an anti-inflammatory agent and in the treatment of malaria. Although its mode of action is unknown, chloroquine is a potent inducer of cellular autophagy. This effect of chloroquine does not appear to be long lasting since the AVs disappear from hepatocytes by 3 weeks after dosing (Abraham et al., 1968).

\section{METHODS AND MATERIALS}

Animals and drug doses. All experiments were carried out on male CFN strain albino rats (100 to $135 \mathrm{~g}$ ) from a randomly inbred colony maintained in this laboratory. The livers from six untreated animals were used as controls. Chloroquine (Aralen hydrochloride, Winthrop Laboratories, N.Y.) was administered intraperitoneally at a dose of $50 \mathrm{mg} / \mathrm{kg}$ body wt. Two animals were decapitated at 1 ,

${ }^{1}$ Supported by the University of Michigan Memorial Phoenix Project Grant 478 and the H. H. Rackham School of Graduate Studies Grant FRF-91. 
2 , and $6 \mathrm{hr}$ after the injection of the drug. A portion of the central lobe of the liver was immediately removed and prepared for electron microscopy.

Electron microscopy. Whole liver samples of approximately $0.5 \mathrm{~mm}^{3}$ were fixed in $3 \%$ glutaraldehyde $-0.1 \mathrm{M}$ sodium phosphate buffer (pH 7.2), for $2 \mathrm{hr}$ at $4^{\circ} \mathrm{C}$. All samples were washed three times in a $0.2 \mathrm{M}$ sucrose $-0.1 \mathrm{M}$ phosphate buffer ( $\mathrm{pH}$ 7.2) and stored overnight at $4^{\circ} \mathrm{C}$. Samples were then postfixed in $2 \% \mathrm{OsO}_{4}-0.1 \mathrm{M}$ phosphate buffer, washed two times in $0.1 M$ phosphate buffer and twice in distilled water, dehydrated through a graded alcohol series and then in propylene oxide, and embedded in Epon. Thin sections were cut with a Reichert-OmU-2 ultramicrotome using a glass or diamond knife and were then placed on Formvar carbon-coated grids. The sections were poststained with $6 \%$ aqueous uranyl magnesium acetate and Reynold's lead citrate. Specimens were examined and photographed with an AEI Corinth 275 electron microscope.

Morphometry. Five blocks of liver tissue, selected randomly, were examined for each animal at two different levels of magnification. Areas selected for micrographs were chosen at random from a single section as described by Weibel et al. (1969). The volume densities of the hepatic and extrahepatic space, as well as the average volume of the hepatocytes were determined from the analysis of at least four negatives, at $1000 \times$, taken of one section from each block. A square-lattice test system containing 100 points and corresponding to a parenchymal area of 2500 $\mu \mathrm{m}^{2}$ was used for the point counting. The number of hepatic nuclei present in the same area was used as an approximation of the number of hepatocytes present in the sample. The volume of cytoplasm associated with each hepatocyte nucleus has been found to be a valid estimation of the actual volume of an average mononuclear hepatocyte (Schmucker et al., 1978).

From a primary magnification of $4000 \times$, at least five photographic prints for every block were enlarged to a final magnification of $20,000 \times$. A second point lattice was overlayed on these prints. It contained 99 points regularly spaced and covering a total area of $99 \mu \mathrm{m}^{2}$ of hepatic tissue. Point counting was then used to evaluate the numerical and volume densities of the AVs, lysosomes, peroxisomes, and mitochondria within the hepatocyte.

The tissue sample was analyzed by the test system through an unbiased, systematic process at every step (Weibel and Bolender, 1973). The photographic fields were selected by using successive corners of the grid squares so that all micrographs at any one magnification were taken from the same thin section. The point lattices were always placed along the same two edges of the negative or micrograph being examined. A total of 240 negatives at the lower magnification and $\mathbf{3 0 0}$ micrographs at the higher magnification were evaluated in this study.

The point or intersection counts were averaged for each block at both magnifications. These numbers were then averaged for each animal and finally for the group of animals at each drug exposure time and for the control group. The calculations were carried out according to Weibel et al. (1969) to yield average values for the following: the organelle volume $\left(\mu \mathrm{m}^{3}\right)$, the number of organelles per hepatocyte, and the hepatocyte volume. All of the stereological data were subjected to statistical computation to determine the standard error and the $95 \%$ confidence interval.

The Holmes effect (Weibel and Bolender, 1973) must be considered as a possible systematic error that occurs in studies such as this. This effect is caused by the fact that the thin sections have a finite thickness but are assumed to be two 
dimensional for the purposes of stereological evaluations. The result is that the area of opaque structures, such as the cytoplasmic components described in this paper, tend to be overestimated to a small degree at the expense of the translucent components present. A correction factor is unnecessary because all parameters considered are assumed to be overestimated to the same extent since the translucent components were not measured.

Organelle identification. Recognition of mitochondria and peroxisome profiles in electron micrographs can be made with little difficulty. However, differentiating lysosomes from AVs involves some degree of subjective judgement. Profiles scored as lysosomes consisted of those typically characterized as primary lysosomes.

No distinction was made between autophagic vacuoles containing recognizable cytoplasmic organelles and particulate components (e.g., glycogen) or those containing more poorly defined or degraded cytoplasmic remnants and membranes. A requirement for a profile to be scored as an AV was that it be clearly separated from the surrounding cytoplasm by its limiting membranes. Similarly, no attempt was made to quantitate the observed ultrastructural changes with respect to specific hepatic areas. Although such information is necessary in some studies, the purpose of this work was to evaluate significant changes in the organelles of the liver as a whole, over time, in treated vs untreated animals.

\section{RESULTS}

The AV is the organelle of primary interest in this study. Examples of the induced organelles are shown in Fig. 1. As the data in Fig. 2A show, chloroquine administration potently induced these organelles within $1 \mathrm{hr}$. The number of $\mathrm{AV}$ per hepatocyte peaked at $2 \mathrm{hr}$ after the drug administration, reaching more than 600 times the control value. The volume per AV also reached a maximum at this time. By $6 \mathrm{hr}$ after drug treatment, both the number and volume of this organelle were beginning to decline from the 2-hr values, however, AVs still outnumbered those present in controls by more than 500 -fold. The average AV volume is not statistically significant in this group because of the limited number of AVs in the hepatocytes from control animals.

Very little change was seen in the number of lysosomes during the first hour after chloroquine administration and only a small increase was evident after a 2 -hr period (Fig. 2B). By $6 \mathrm{hr}$, however, the number of lysosomes per cell decreased by about $40 \%$ as compared with that of the controls. The average volume of the lysosomes, however, decreased at 1 and $2 \mathrm{hr}$ followed by a return to near control values by $6 \mathrm{hr}$ (Fig. 2B).

The hepatocyte peroxisome exhibited the second largest change in number. The reduction in the average number of peroxisomes per hepatocyte at all three time periods is illustrated in Fig. 2C. At $2 \mathrm{hr}$, an $80 \%$ decrease in the number of peroxisomes was concomitant with an $82 \%$ increase in the volume per organelle as compared with control values. By the sixth hour, the average peroxisome volume declined, but was still $30 \%$ over control values. The number of peroxisomes at 6 hours was still $80 \%$ below that found in control hepatocytes.

The number of mitochondria appeared to be affected by the chloroquine treatment to a lesser extent than did the numbers of AVs or peroxisomes (Fig. 2D). At $1 \mathrm{hr}$ there was about a $10 \%$ increase in the number and volume of mitochondria. 

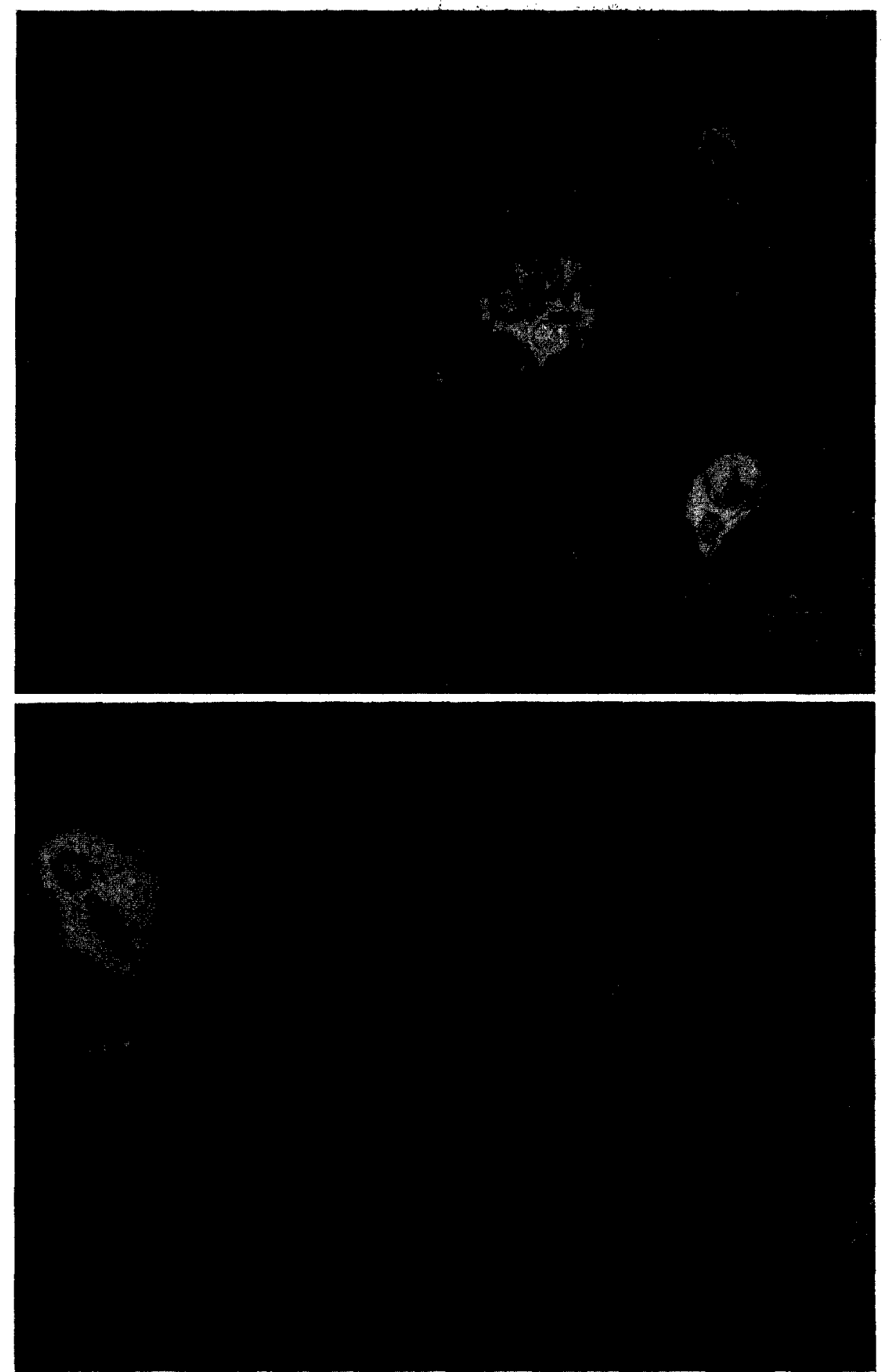

FIG. 1. Portions of rat hepatocytes $2 \mathrm{hr}$ following chloroquine administration. N, nucleus; bc, bile canaliculus; $M$, mitochondria; $P$, peroxisome; arrowheads, autophagic vacuoles. (A) AVs with unidentified contents, $13,000 \times$. (B) AVs with identifiable mitochondria $\left({ }^{*}\right)$ and peroxisome $\left({ }^{*}\right), 32,000 \times$. 

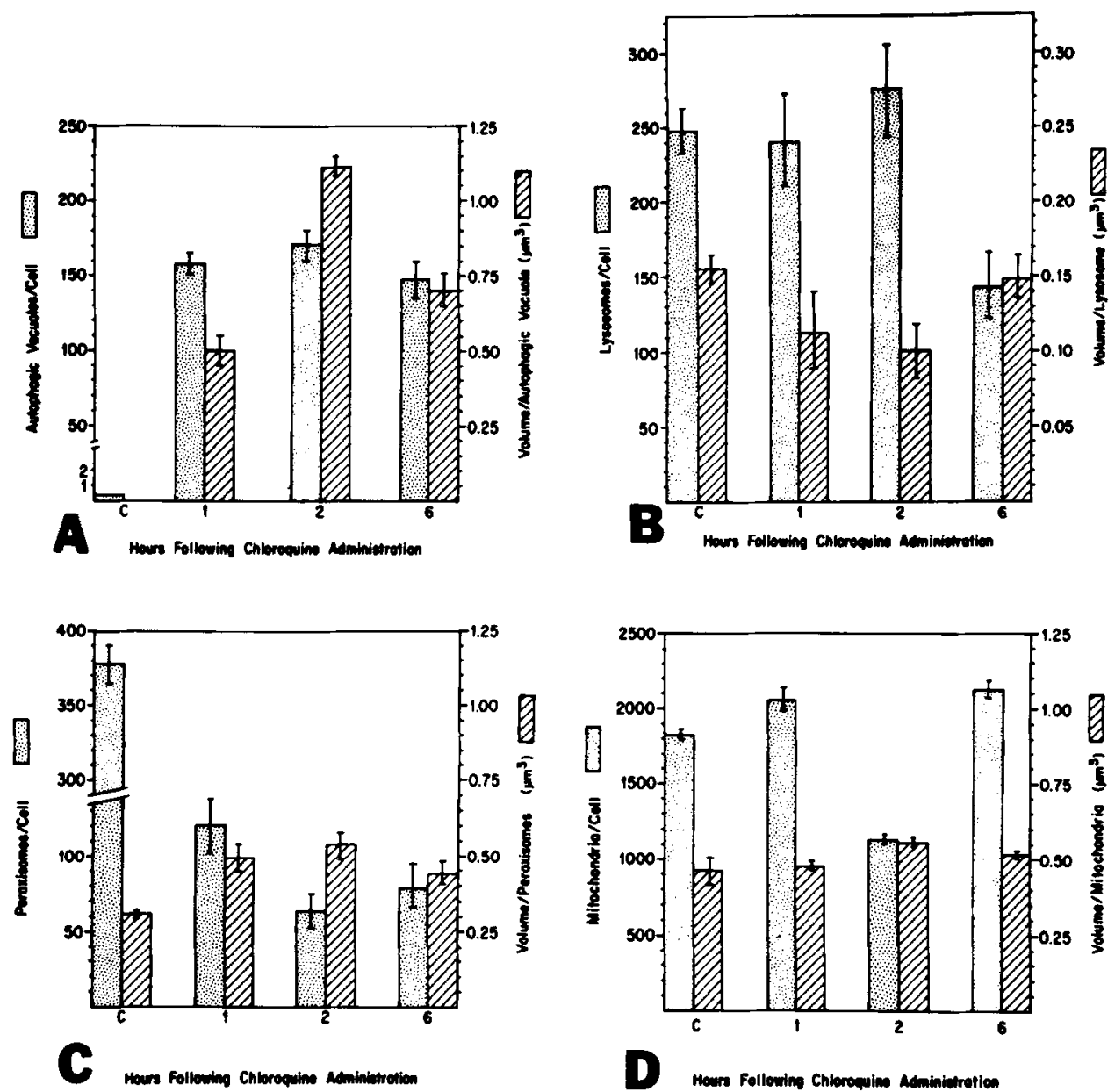

Fig. 2. Average organelle number and volume per mononuclear hepatocyte. The bars represent the 95\% confidence levels of each parameter. Volume for the control AV is not included since the standard error is greater than the mean because of the limited number of these organelles in the control hepatocytes.

Two hours after chloroquine administration the number of mitochondria decreased to about $62 \%$ of the number found in the control hepatocytes. At $6 \mathrm{hr}$ the average number of mitochondria was about $17 \%$ over the controls. The average mitochondria volumes at 2 and 6 hours were about $30 \%$ over those of the controls.

The average hepatocyte volume decreased after both 1 and $2 \mathrm{hr}$, and by $6 \mathrm{hr}$ it approached control values. The ratios between experimental hepatocytes and organelle volumes and the respective volumes observed in control hepatocytes are shown in Fig. 3. The ratios between hepatocyte cell volumes closely paralleled the pattern for the ratios observed between lysosome volumes. The ratios of the mitochondria and peroxisome volumes were greater than 1 at each time period and reached a peak at $2 \mathrm{hr}$.

Figure 4 shows the number of organelles per hepatocyte expressed as the ratio between the experimental and the control values. At $2 \mathrm{hr}$, the AVs showed the greatest increase in number while the peroxisomes showed the greatest decrease 
in number. The number of mitochondria initially increased, then declined at $2 \mathbf{~ h r}$ and by $6 \mathrm{hr}$ had increased to slightly greater than the control value. Lysosomes showed a pattern which was reciprocal to that of the mitochondria.

Table I illustrates the total organelle volume per hepatocyte and the fractional volume (in percent) of the organelle per hepatocyte. The chloroquine induction of AVs was evident here with a peak at $2 \mathrm{hr}$ for both the total volume and the fractional percentage of AVs per hepatocyte. The fractional volume of lysosomes per hepatocyte also showed a peak at $2 \mathrm{hr}$ while the total volume steadily decreased through the sixth-hour period. Peroxisomes showed a continual decline in total volume and the hepatocyte fractional volume between 1 and $6 \mathrm{hr}$. The total volume of mitochondria showed a fluctuation with time but the fractional volume of mitochondria was about $50 \%$ above the control values during the 6 -hr period.

\section{DISCUSSION}

The use of stereological methods to sample tissues provides a numerical basis for evaluating subcellular changes in experimental tissues as compared with that of controls. The results clearly show that the frequency with which AVs appear in hepatic tissue can be significantly and rapidly increased following the in vivo administration of the drug chloroquine. Other membrane-bound organelles are also shown to undergo significant changes in their average volumes, and their average numbers per cell at 1,2, and $6 \mathrm{hr}$ after treatment with the drug. The data illustrate that the maximum effect observed, whether an increase or decrease in organelle number or volume, occurs most frequently at $2 \mathrm{hr}$ following administration of the drug.

The volume per AV peaks at 2 hr (Fig. 2A) following drug administration, as does the fractional volume of AV per hepatocyte (Table I). Both of these parameters are beginning to decrease by 6 hours after treatment. It appears as though the chloroquine-induced autophagy has reached its maximum, at least in terms of cell volume, well within the first $6 \mathrm{hr}$ after stress. The number of AVs per hepatocyte is rapidly induced. This induction is evident after only $1 \mathrm{hr}$ and increases slightly by $2 \mathrm{hr}$. Between 2 and $6 \mathrm{hr}$ there seems to be a turning point at which the hepatocytes, by mechanisms yet unknown, begin to return to the control levels.

Both the mitochondria and the peroxisomes show a reduction in number at $2 \mathrm{hr}$. Both types of organelles have been observed within the AV (Fig. 1B). However, the frequency of recognizable mitochondria within AV seems to outnumber by far the number of peroxisomes observed in the early forming AVs. Since it is not always possible to identify the sequestered components within the $A V$, it is not possible to determine whether the induced AV's are responsible for the decline in the number of mitochondria. However, the number of mitochondria per cell increases above control values by $6 \mathrm{hr}$. Therefore, the greatest reduction in the number of this organelle occurs within the first $2 \mathrm{hr}$, which corresponds with the time that the number and volume of AVs are rapidly increasing.

The rapid decline in the number of peroxisomes is not believed to be due solely to the sequestration by the AV. This decrease in the number of peroxisomes is accompanied by an increase (about $82 \%$ ) in volume. It is not known whether this enlarged state is an effect or a result of the induced stress and subsequent accommodation. Chloroquine, however, is known to be a membrane antagonist (Ericsson, 1969) and the decrease in the number of peroxisomes, as well as their 


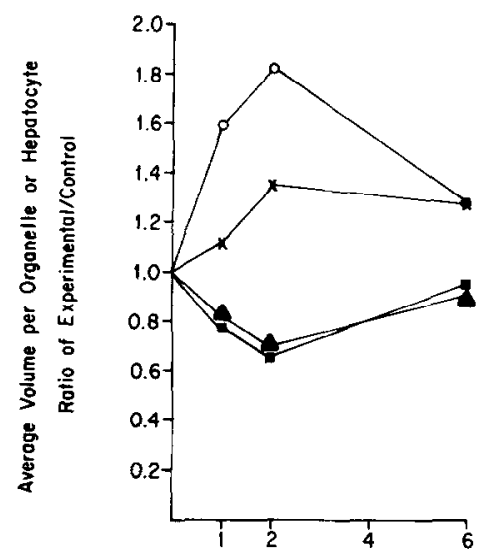

(3)

Hours following Chloroquine Administration

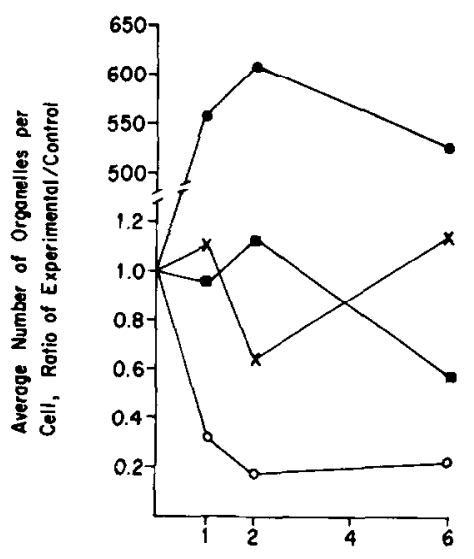

(4)

Hours Following Chloroquine Administration

FIG. 3. Ratio of organelle or hepatocyte cell volume between experimental and control animals. $(O)$ peroxisome, ( $\mathbf{(})$ lysosome, (x) mitochondria, (A) hepatocytes.

FIG. 4. Ratio of organelle number per hepatocyte between experimental and control animais. AV, (O) peroxisome, (D) lysosome, (x) mitochondria.

increase in size, suggests that the organelle is undergoing lysis as well as being sequestered by the AV. A decreased electron density is observed in the enlarged peroxisome profiles. Deter (1971) also reported a reduction in the number of peroxisomes in hepatocytes after treatment with glucagon, a potent AV inducer.

The lysosomes increase in number by the second hour, but are smaller in size. This may reflect lysosome biogenesis. By the sixth hour, lysosomes show a decrease of more than $40 \%$ in number per cell as compared to that of the 2 -hr and control values. This decrease could be explained as a result of fusion of lysosomes with the AVs. Ericsson (1969) has postulated that lysosomes fuse with and transfer their acid hydrolases to AVs. Such an event would support the finding of lysosomes of smaller volume, since it is possible to section through a lysosome, fused with an AV and partially emptied, without seeing the attached AV in that section plane. Our results are consistent with this hypothesis since the volume of lysosomes initially decreases at 1 and $2 \mathrm{hr}$ and then increases to about the control level at $6 \mathrm{hr}$. The average volume of the lysosomes at 6 hours is very close to that

\section{TABLE I}

Organelle Volume $\left(\mu \mathrm{m}^{3}\right)$ per Hepatocyte and the Percentage Fraction Volume of Hepatocyte Occupied by Organelle

\begin{tabular}{|c|c|c|c|c|c|c|c|c|}
\hline \multirow[b]{2}{*}{ Organelle } & \multicolumn{2}{|c|}{ Control } & \multicolumn{2}{|c|}{$1 \mathrm{hr}$} & \multicolumn{2}{|c|}{$2 \mathrm{hr}$} & \multicolumn{2}{|r|}{$6 \mathrm{hr}$} \\
\hline & $\begin{array}{c}\text { Total } \\
\text { vol } \\
\left(\mu \mathrm{m}^{3}\right)^{\alpha}\end{array}$ & $\begin{array}{c}\text { Percentage } \\
\text { of } \\
\text { hepatocyte }^{b}\end{array}$ & $\begin{array}{c}\text { Total } \\
\text { vol } \\
\left(\mu \mathrm{m}^{3}\right)^{a}\end{array}$ & $\begin{array}{c}\text { Percentage } \\
\text { of } \\
\text { hepatocyte }^{b}\end{array}$ & $\begin{array}{c}\text { Total } \\
\text { vol } \\
\left(\mu \mathrm{m}^{3}\right)^{a}\end{array}$ & $\begin{array}{c}\text { Percentage } \\
\text { of } \\
\text { hepatocyte }^{b}\end{array}$ & $\begin{array}{c}\text { Total } \\
\text { vol } \\
\left(\mu \mathrm{m}^{3}\right)^{a}\end{array}$ & $\begin{array}{c}\text { Percentage } \\
\text { of } \\
\text { hepatocyte }^{b}\end{array}$ \\
\hline AV & 0.38 & 0.001 & 78.5 & 2.31 & 195 & 7.13 & 103 & 2.85 \\
\hline Lysosome & 38.3 & 0.92 & 28.9 & 0.85 & 27.5 & 1.01 & 21.2 & 0.59 \\
\hline Peroxisome & 117 & 2.91 & 58.8 & 1.70 & 35.8 & 1.31 & 31.4 & 0.87 \\
\hline Mitochondria & 728 & 18.1 & 924 & 27.3 & 712 & 26.0 & 1102 & 30.5 \\
\hline
\end{tabular}

a Total volume is the average volume/organelle times the average number of organelles/hepatocyte.

- Percentage of hepatocyte is normalized to the corresponding average hepatocyte volume. 
in the control hepatocytes although the total lysosomal volume per hepatocyte is still low, primarily as a result of the decrease in the number of this organelle. If lysosomes are fusing with AVs, biogenesis of new lysosomes would be expected to occur, which could also account for the smaller volume of "new" lysosomes seen at 1 and $2 \mathrm{hr}$.

By the sixth hour the morphometric data show that the average AV volume and number have declined from the peak at $2 \mathrm{hr}$. At $6 \mathrm{hr}$ there are very few recognizable organelles observed within the AVs. The reduction in volume seems to parallel the digestion of identifiable contents within the early forming AVs into the mostly unrecognizable contents seen at $6 \mathrm{hr}$. The number of peroxisomes present at $6 \mathrm{hr}$ is still only $21 \%$ of the number found in the control. These results differ from those of Abraham et al. (1968), who reported that these organelles were uniform in shape and size but "showed an appreciable increase in number" over that of the control. The average volume of peroxisomes decreases between 2 and $6 \mathrm{hr}$, thus approaching that of the control level. This would seem to indicate that the effect of the drug which caused the increase in peroxisome size is diminishing. Even though the individual peroxisomes are larger than those seen in controls, the total and fractional volumes of this organelle per hepatocyte are still well below those of the control value (Table I). This can be explained by the sharp decrease in the number per cell, which shows only slight recovery at $6 \mathrm{hr}$ as compared with that present at 2 hr (Fig. 2C).

The average volume of the hepatocyte mitochondria shows less variability than any other parameter analyzed (Fig. 2D). At $6 \mathrm{hr}$, the average number of mitochondria per cell has nearly doubled since the 2 -hr period. This increase may reflect the biogenesis of mitochondria after many have been sequestered by the autophagic process.

These studies have demonstrated the quantitative changes in organelle number per hepatocyte and in their average volume before and after chloroquine treatment. The greatest changes occur in ultrastructural parameters at $2 \mathrm{hr}$ after administration of the drug, except in the case of lysosomes, in which the greatest deviation in number occurs at $6 \mathrm{hr}$. This study can be used as a model for druginduced autophagy. Further studies on AVs during the peak induction period will aid in determining whether this process is important in the accommodation of stress.

\section{ACKNOWLEDGMENTS}

The authors wish to acknowledge the assistance of Mr.,David Parker and Mr. Richard P. Thomas for computer programming this work, and Ms. Penelope Pietras for typing the manuscript.

\section{REFERENCES}

Abraham, R., Hendy, R., and Grasso, P. (1968). Formation of myeloid bodies in rat liver lysosomes after chloroquine administration. Exp. Mol. Pathol. 9, 212-229.

Deter, R. L. (1971). Quantitative characterization of dense body, autophagic vacuole, and acid phosphate-bearing particle populations during the early phases of glucagon-induced autophagy in rat liver. J. Cell Biol. 48, 473-489.

Ericsson, J. L. (1969). Mechanism of cellular autophagy. In "Lysosomes in Biology and Pathology" (J. T. Dingle and H. B. Fell, eds.), Vol. 2, pp. 345-394. Wiley, New York.

Holtzman, E. (1973). "Lysosomes: A Survey." Springer-Verlag, New York.

Leedale, G. F., and Buetow, D. E. (1976). Observations on cytolysome formation and other cytological phenomena in carbon-starved Euglena gracilis. J. Microsc. Biol. Cell. 25, 149-154.

Schmucker, D. L., Mooney, J. S., and Jones, A. L. (1978). Stereological analysis of hepatic fine 
structure in the Fischer 344 rat. influence of sublobular location and animal age. J. Cell Biol. 78, $319-337$.

Weibel, E. R., Staubli, W., Gnagi, H. R., and Hess, F. A. (1969). Correlated morphometric and biochemical studies on the liver cell. I. Morphometric model, stereological methods and normal morphometric data for rat liver. J. Cell Biol. 42, 68-91.

WEIBEL, E. R., and BOLENDER, R. P. (1973). Stereological techniques for electron microscopic morphometry. In "Principles and Techniques of Electron Microscopy" (M. A. Hayat, ed.), Vol. 3, pp. 237-296. Reinhold, New York.

Wisner-Gebhart, A. M., Brabec, R. K., and Gray, R. H. (1979). Morphometric studies of chloroquine induced autophagy in rat liver. Micron 10, 65-66. 